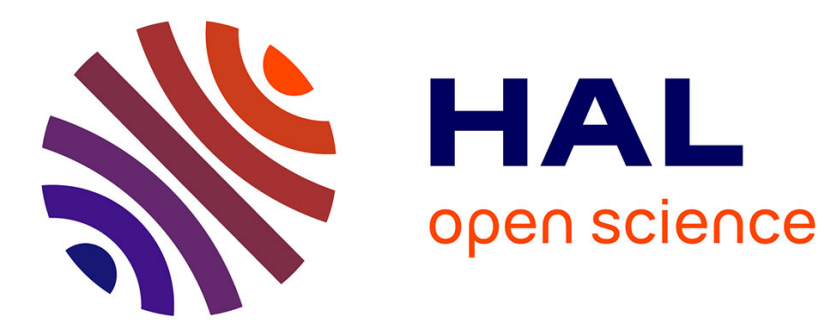

\title{
Charge density and plasmon modes in a triangular quantum well model for doped and undoped gated AlGaN/GaN HEMTs
}

S Rabbaa, J Stiens

\section{- To cite this version:}

S Rabbaa, J Stiens. Charge density and plasmon modes in a triangular quantum well model for doped and undoped gated AlGaN/GaN HEMTs. Journal of Physics D: Applied Physics, 2011, 44 (32), pp.325103. 10.1088/0022-3727/44/32/325103 . hal-00642389

\section{HAL Id: hal-00642389 \\ https://hal.science/hal-00642389}

Submitted on 18 Nov 2011

HAL is a multi-disciplinary open access archive for the deposit and dissemination of scientific research documents, whether they are published or not. The documents may come from teaching and research institutions in France or abroad, or from public or private research centers.
L'archive ouverte pluridisciplinaire HAL, est destinée au dépôt et à la diffusion de documents scientifiques de niveau recherche, publiés ou non, émanant des établissements d'enseignement et de recherche français ou étrangers, des laboratoires publics ou privés. 


\title{
Charge density and plasmon modes in a triangular quantum well model for doped and undoped gated AlGaN/GaN HEMTs
}

\author{
S Rabbaa and J Stiens \\ Laboratory of Micro- and PhotonElectronics, Dept. of Electronics and Informatics, \\ Vrije Universiteit Brussel, Pleinlaan 2, 1050-Brussels, Belgium. \\ E-mails: srabbaa@etro.vub.ac.be ; jstiens@etro.vub.ac.be
}

\begin{abstract}
We have calculated the plasmon frequency of the two-dimensional electron gas (2DEG) in AlGaN/GaN high electron mobility transistors (HEMT). The impact of HEMT's parameters on the plasmon frequency and the sheet charge density of the 2DEG is discussed in detail. The charge density in the HEMT's channel is calculated by means of a triangular quantum well (TQW) model. It has been found that the $\mathrm{AlGaN} / \mathrm{GaN}$ heterostructure induces plasmon oscillations in the $\mathrm{THz}$ range with larger frequencies compared to other semiconductor compounds. The sensitivity of the tunability of these frequencies is considerable, especially by using a variable applied gate voltage. We have derived optimal structure parameters for obtaining a maximum plasmon frequency for a given doping concentration. We will show that the accuracy of this optimized frequency value is dependent on the average position $\Delta \mathrm{d}$ of charge density in the triangular shaped (quantum well) channel. The interaction between radiation and plasmons has many applications such as detectors, mixers and generators of $\mathrm{THz}$ waves.
\end{abstract}

Keywords: Plasmon frequency, 2DEG, HEMT, AlGaN/GaN heterostructure, sheet charge density, THz.

\section{Introduction}

During the last decade of the $21^{\text {th }}$ century, considerable progress had been made in the technology of gallium nitride $(\mathrm{GaN})$ based HEMTs. GaN has unique properties that make it an attractive electronic and optoelectronic material. It is optically transparent and it has exceptional chemical and physical stability. It is used in biosensors fabrication due to its properties of non- toxic surface content (like As) and the immobilization of bio-molecules [1]. The AlGaN/GaN based HEMTs have some advantages that make it outperform upon other heterostructures. Some of these features are: introducing high density 2DEG, high current density, high carrier mobility, low sheet resistance and high transconductance [2]. The sheet charge density can become an order of magnitude larger (as we will see) than in GaAs/AlGaAs heterostructures which provides a plasmon frequency about 3 times larger.

The frequency response of the two-dimensional (2D) electron channel in HEMTs is affected by the excitation of plasma oscillations of the 2DEG under the gate electrode [3]. The coupling mechanism between 2D plasmons and external electromagnetic waves were discussed in [4-7]. It was suggested that this coupling might be stronger using the design of field effect transistors array with grating gates [8]. This excitation mechanism allows for the design of $\mathrm{THz}$ tunable detectors and generators. The plasmon oscillation in 2D promotes effective emission of $\mathrm{THz}$ radiation [8]. The use of $\mathrm{THz}$ spectrum in the sensing of chemical and biological systems and in the detection of hidden weapons and mines requires high sensitive and fast response detectors. THz emitters and detectors are used in medical, biological and industrial imaging, communications and space sciences [1,9]. The plasmon frequency of the 2DEG in AlGaN/GaN based HEMT belongs to the THz gap and it can exceed $10 \mathrm{THz}$ 
compared to the low band of $\mathrm{THz}$ frequency range in conventional classical semiconductor devices such as tunneling diodes and classical transistors [10].

After giving a short overview of the various expressions of the plasmon frequency in various (gated) structures and its relation to the charge density, our structure is introduced in section 3 with a description of the induced polarizations in its channel. Our focus is laid upon the design parameters and the analysis of TQW in section 4. Calculations and discussions are presented in section 5.

\section{Plasmon Frequency in 2DEG System}

The frequency of plasmon oscillations in three-dimensional (3D) materials is given by:

$$
\omega_{p}^{2}=\frac{e^{2} N}{\varepsilon_{o} \varepsilon_{\infty} m^{*}} \quad\left(\mathrm{rad}^{2} / \mathrm{s}^{2}\right)
$$

with $\mathrm{N}$ the free carrier concentration, e the electron charge, $\mathrm{m}^{*}$ the electron effective mass, $\varepsilon_{\infty}$ the high frequency dielectric constant and $\varepsilon_{\mathrm{o}}$ the vacuum permittivity. The phonon-plasmon coupling and the interaction between plasmons and $\mathrm{THz}$ radiation in bulk GaN was discussed in [1]. The 3D plasmon frequency in highly doped $\mathrm{GaN}$ has $\mathrm{THz}$ applications even in the range of $\mathrm{CO}_{2}$ laser radiation.

Plasmon oscillations in 2D electron system were introduced by Stern [11] and they were observed experimentally in a sheet of electrons in liquid helium [12] and in silicon metal-insulatorsemiconductor structures [13]. There are two types of excited plasma waves in HEMTs: gated (screened) plasmons and un-gated (unscreened) plasmons. The spectrum of the gated plasmon modes which are excited under the gate contact is given by:

$$
\omega_{p}^{2}=\frac{e^{2} N_{s} k}{m^{*} \varepsilon_{o}\left[\varepsilon_{s}+\varepsilon_{b} \operatorname{coth}(k d)\right]} \quad\left(\operatorname{rad}^{2} / \mathrm{s}^{2}\right)
$$

where $\mathrm{N}_{\mathrm{s}}$ is the sheet carrier concentration at the heterostructure interface, $\mathrm{k}$ is the plasmon wavevector, $\varepsilon_{\mathrm{s}}, \varepsilon_{\mathrm{b}}$ are the dielectric constants of surrounding materials (substrate and barrier materials, respectively), and $\mathrm{d}$ is the distance between gate plane and the $2 \mathrm{D}$ electron channel [8]. The wavevector is given by $k=\pi n /(L+2 d)$, where $n=1,2,3 \ldots$, and $L$ is the gate length. When $n$ is even, plasmon modes of the charge density under the gate cannot be excited with $\mathrm{THz}$ radiation due to zero net dipole moment of the modes. Therefore, to get odd modes, the wavevector should be taken as $\mathrm{k}=$ (2n-1) $\pi / \mathrm{L}$. If $\mathrm{kd}<<1$, 'equation 2' can be written as:

$$
\omega_{p}^{2}=\frac{e^{2} N_{S} d}{\varepsilon_{o} \varepsilon_{b} m^{*}} k^{2}
$$

If the barrier layer is thin compared to the gate length $(\mathrm{d}<<\mathrm{L})$, then the first harmonic of plasmon oscillations with $\mathrm{n}=1$ (the fundamental plasmon mode) can be given in terms of the gate voltage $\left(\mathrm{V}_{\mathrm{g}}\right)$ and threshold voltage $\left(\mathrm{V}_{\mathrm{th}}\right)$ as:

$$
\omega_{p}^{2}=\frac{e\left(V_{g}-V_{t h}\right)}{m^{*}} k^{2}
$$

Although it may be possible to achieve large THz plasmon frequencies by decreasing the gate length, there are actually some limitations in designing HEMTs with very short gate lengths. According to 'equation 4', the modes excited under the gate electrode can be tuned by changing $\mathrm{V}_{\mathrm{g}}$, and so they have more practical applications than un-gated modes [8].

\section{Induced polarization in AlGaN/GaN heterostructure}

A cross section of a HEMT is shown in 'figure 1'. The epitaxial layers are usually grown by various techniques such as molecular beam epitaxy (MBE), Metalorganic vapor phase epitaxy (MOVPE) and metalorganic chemical vapor deposition (MOCVD) on a substrate, typically 
sapphire or $\mathrm{SiC}$. The growth sequence is: undoped $\mathrm{GaN}$ buffer layer of thickness $0.6-3 \mu \mathrm{m}$ (typically $1 \mu \mathrm{m}$, followed by undoped i-AlGaN spacer layer of thickness $d_{i}$ between $0-10 \mathrm{~nm}$, and finally a doped $\mathrm{AlGaN}$ layer of thickness $\mathrm{d}_{\mathrm{n}}$. The purpose of the spacer layer is to reduce Coulombic scattering caused by electrical interaction between the electrons in the 2DEG and ionized donors in the doped AlGaN layer, which will increase the electron mobility [14]. A 2DEG of average position $\Delta \mathrm{d}$ from the heterointerface is formed at the heterostructure of the buffer and spacer layers.

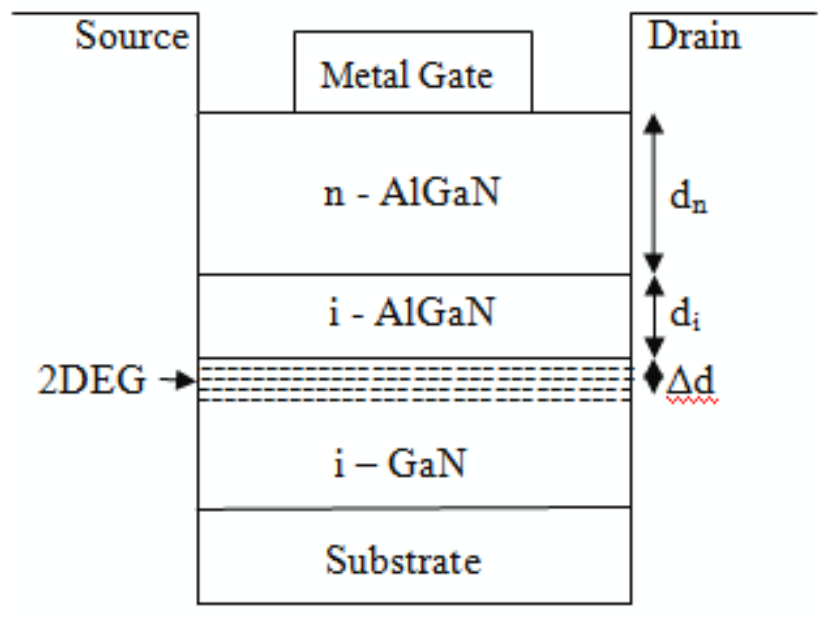

'Figure 1': Schematic diagram of AlGaN/GaN HEMT structure.

A high charge is induced in the channel even if the upper layer of $\mathrm{AlGaN}$ is undoped or/and without external electric field $\left(\mathrm{V}_{\mathrm{g}}=0\right)$. This is one of the $\mathrm{AlGaN} / \mathrm{GaN}$ heterostructure properties and advantages over other heterojunctions. In this case there are two types of macroscopic polarizations [15]:

- Spontaneous polarization $\left(P_{s p}\right)$ : due to the cation and anion positions in the lattice. It is given in terms of the Al-mole fraction $(x)$ as:

$$
P_{s p}=-0.090 x-0.034(1-x)+0.019 x(1-x) \quad\left(\mathrm{C} / \mathrm{m}^{2}\right)
$$

- Piezoelectric polarization $\left(P_{p z}\right)$ : due to lattice mismatch between $\mathrm{GaN}$ and $\mathrm{AlGaN}$ layers. It is given using Vegard's law by:

$$
P_{p z}^{A l G a N}=x P_{p z}^{A l N}+(1-x) P_{p z}^{G a N}
$$

where $P_{p Z}^{A l N}$ and $P_{p Z}^{G a N}$ are given in terms of the strain $\in$ as:

$$
\begin{gathered}
P_{p z}^{A l N}=-1.808 \in-7.888 \epsilon^{2} \\
P_{p z}^{G a N}=-0.918 \in+9.541 \epsilon^{2}
\end{gathered}
$$

Nitride wafers are generally grown along the c-axis, and as a result the strain exits onto the epitaxial layer in the basal plane, and it is given by: 


$$
\in(x)=\frac{a_{o}-a(x)}{a(x)}
$$

where $\mathrm{a}(x)=0.31986-0.00891 x$ is the lattice constant of $\mathrm{AlGaN}$, and $\mathrm{a}_{0}=\mathrm{a}(x=0)$ is the lattice constant of $\mathrm{GaN}$.

The above expressions give the two polarizations as a quadratic equation of $x$ (nonlinear polarization). It was found experimentally $[15,16]$ that the nonlinear model is more accurate than linear ones which were considered in some research works.

\section{The sheet charge concentration}

The total polarization $(\sigma)$ induced at the heterostructure is related to $P_{s p}$ and $P_{p z}$ of the two layers forming the heterostructure by the following equation [17]:

$$
\sigma(x)=P_{p Z}^{A l G a N}+P_{s p}^{A l G a N}-P_{s p}^{G a N}
$$

where we consider $P_{p Z}^{G a N} \approx 0$ because its thickness is much larger than the strained AlGaN layer, and so it is fully relaxed. The free charge carriers are assumed approximately to occupy a triangular quantum well which contains a constant built-in electric field $[18,19]$. To find the energy states and the population of free electrons in each energy state, we have solved Schrodinger's equation for the TQW. A detailed solution can be found in [20]. The energy eigenvalues are given by:

$$
E_{j}=\left(\frac{\hbar^{2}}{2 m^{*}}\right)^{1 / 3}\left[\frac{3 \pi e \mathcal{F}_{e f f}}{2}\left(j+\frac{3}{4}\right)\right]^{2 / 3} \quad \mathrm{j}=0,1,2 \ldots
$$

We are interested in the first two energy states: $\mathrm{E}_{0}$, the ground state, and $\mathrm{E}_{1}$, the first excited state. For undoped or lowly doped heterostructures, $\mathcal{F}_{\text {eff }}=\mathcal{F}_{S}$, while for highly doped case, the effective electric field is $\mathcal{F}_{\text {eff }}=\mathcal{F}_{S} / 2$, where the surface field $\mathcal{F}_{S}=e N_{s} / \varepsilon_{s}$, with $\varepsilon_{\mathrm{s}}$ the dielectric constant of $\mathrm{GaN}$ and $\mathrm{N}_{\mathrm{s}}$ the charge density at the heterostructure given by [20]:

$$
N_{S}=D k_{B} T \sum_{j=0}^{1} \ln \left[1+\exp \left(\frac{E_{F-} E_{j}}{k_{B} T}\right)\right]=D k_{B} T \ln \left\{\left[1+\exp \left(\frac{E_{F-} E_{0}}{k_{B} T}\right)\right]\left[1+\exp \left(\frac{E_{F-} E_{1}}{k_{B} T}\right)\right]\right\}
$$

where $\mathrm{D}=\mathrm{m}^{*} / \pi \hbar$ is the conduction band density of states of the $2 \mathrm{D}$ system, $\mathrm{k}_{\mathrm{B}}$ is Boltzmann's constant, $\mathrm{T}$ is the absolute temperature and $\mathrm{E}_{\mathrm{F}}$ is the Fermi energy.

When two different semiconductors (like $\mathrm{GaN}$ and $\mathrm{AlGaN}$ ) form a heterostructure, charges transfer across the interface until the Fermi energy on both sides are equal, because the surface depletion layer and heterojunction interface depletion regions overlap. Therefore, electrons are transferred from the ionized donors in doped AlGaN layer to the conduction band. Thus, the GaN layer at the interface is populated by transferred charges from the barrier and the polarization induced charged at the heterointerface as discussed in section 3. If we assume complete depletion of the AlGaN barrier, taking into account the polarization effect and the applied gate potential, then the charge density concentration at the $\mathrm{AlGaN} / \mathrm{GaN}$ heterointerface can be obtained by solving the one dimensional Poisson's equation, and it is given as [21,22]:

$$
N_{s}=\frac{\varepsilon(x)}{e\left(d_{n}+d_{i}+\Delta d\right)}\left(V_{g}-V_{t h}-V_{c}-\frac{E_{F}}{e}\right)
$$


where $\varepsilon(x)=9.5-0.5 x$ is the dielectric constant of $\mathrm{Al}_{x} \mathrm{Ga}_{1-\mathrm{x}} \mathrm{N}, \mathrm{V}_{\mathrm{c}}$ is the channel potential at a certain distance from the source due to drain source potential, and it can be obtained in [23]. The 2DEG average position $\Delta \mathrm{d}$ from the heterointerface can be calculated from the weighted average position of all energy levels [24]:

$$
\Delta d=\frac{\frac{2}{3} \sum_{j} N_{s j} d_{j}}{N_{s}}
$$

where $\mathrm{N}_{\mathrm{sj}}$ and $\mathrm{d}_{\mathrm{j}}$ are the charge density and average distance of carriers in the $\mathrm{jth}$ subband, respectively, and $\mathrm{N}_{\mathrm{s}}$ is the total carrier concentration. Using Fermi-Dirac statistical functions to determine the population charge $\mathrm{N}_{\mathrm{sj}}$, we find that $\mathrm{N}_{\mathrm{so}}$ (ground state) $>\mathrm{N}_{\mathrm{s} 1}$ (first excited state). Thus $\Delta \mathrm{d}=2 \mathrm{E}_{\mathrm{o}} / 3 \mathrm{e} \mathcal{F}_{\text {eff }}[25]$. The threshold voltage is given by:

$$
V_{t h}(x)=\phi_{b}-\frac{\Delta E_{c}}{e}-\frac{e N_{d} d_{n}^{2}}{2 \varepsilon(x)}-\frac{|\sigma(x)|}{\varepsilon(x)}\left(d_{n}+d_{i}\right)
$$

where $\phi_{b}=1.3 x+0.84$ is the Schottky barrier, $\Delta E_{c}=0.7\left[\mathrm{E}_{\mathrm{g}}(x)-\mathrm{E}_{\mathrm{g}}(0)\right]$ is the conduction band discontinuity between $\mathrm{AlGaN}$ and $\mathrm{GaN}$, with $\mathrm{E}_{\mathrm{g}}=6.13 x+3.42(1-x)-\mathrm{x}(1-x)$ is the band gap of $\mathrm{AlGaN}$, and $\mathrm{N}_{\mathrm{d}}$ is the doping concentration in the barrier $\left(\varepsilon(x), \phi_{b}, \Delta E_{c}\right.$, and $\mathrm{E}_{\mathrm{g}}$ are taken from [17]).

\section{Results and discussions}

As it is clear in 'equation 2', the plasmon frequency depends on the HEMT parameters and on the sheet charge density which is a function of the gate voltage. We can control required values of $\mathrm{N}_{\mathrm{s}}$ (and then $\omega_{\mathrm{p}}$ ) by changing the quantities appearing in 'equations 12 and 13', which are: $\mathrm{m}^{*}, \varepsilon$ (they depend on the type of material), $\mathrm{d}_{\mathrm{i}}, \mathrm{d}_{\mathrm{n}}, \mathrm{N}_{\mathrm{d}}, x$ and $\mathrm{V}_{\mathrm{g}}$. Therefore, we have firstly calculated numerically the sheet charge density at different quantities of the previous independent variables. Because we have a function of many variables, then we plot the relationship with one independent variable and all other variables are fixed. All calculations are taken at temperature $\mathrm{T}=300 \mathrm{~K}$, and at spacer layer thickness $\mathrm{d}_{\mathrm{i}}=3 \mathrm{~nm}$ and $\mathrm{V}_{\mathrm{c}}=0$.

Solving 'equations 12 and 13' simultaneously leads to an implicit relationship between $\mathrm{N}_{\mathrm{s}}$ and the other variables. 'Figure 2' shows the relationship between charge density and gate voltage for doped and undoped heterostructures. The curves consist of two parts: the first is at $\mathrm{Vg}<\mathrm{V}_{\text {th }}\left(\mathrm{N}_{\mathrm{s}}=0\right)$ and the second is at $\mathrm{Vg}>\mathrm{V}_{\text {th }}$ (inclined lines). The slope of each line is related to the capacitance of the structure. For the doped case, the slopes are $2.42 \times 10^{12} \mathrm{~cm}^{-2} \mathrm{~V}^{-1}, 1.95 \times 10^{12} \mathrm{~cm}^{-2} \mathrm{~V}^{-1}$ and $1.66 \times 10^{12} \mathrm{~cm}^{-}$ ${ }^{2} \mathrm{~V}^{-1}$ at $\mathrm{d}_{\mathrm{n}}=15,20$, and $25 \mathrm{~nm}$, respectively. We notice that the slopes, and then the capacitance, are
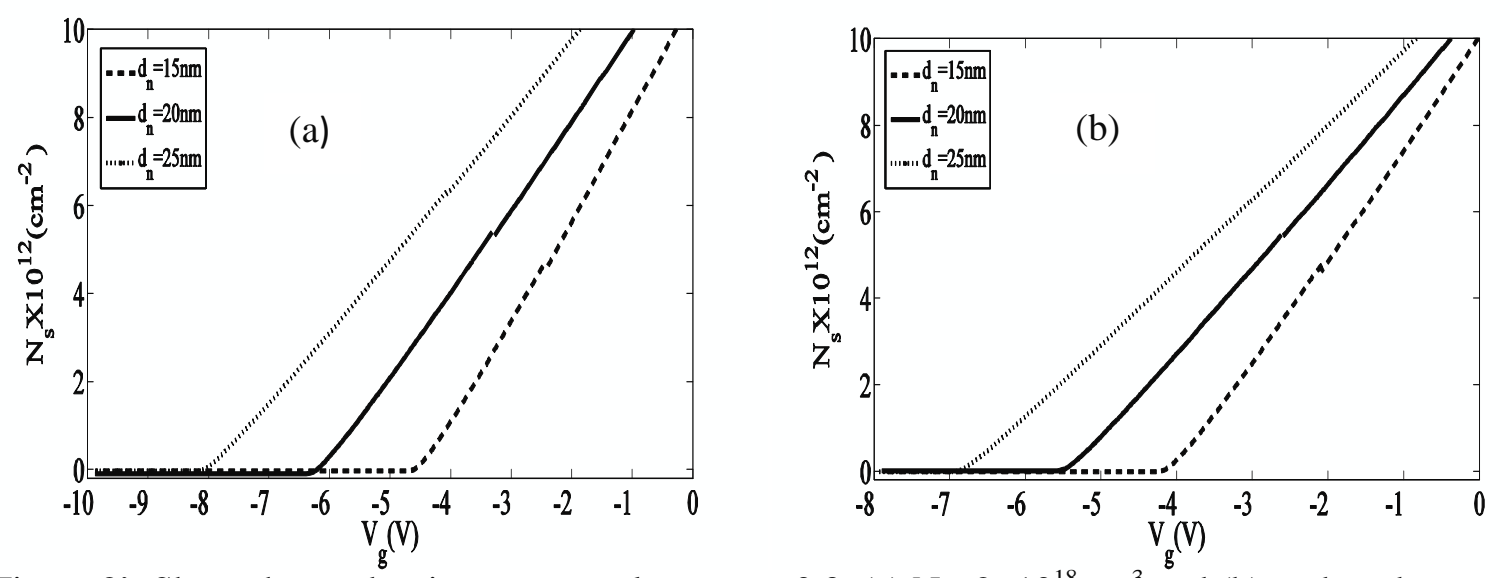

'Figure 2': Sheet charge densitv vs gate voltage at $\mathrm{x}=0.3$. (a) $\mathrm{N}_{d}=2 \times 10^{18} \mathrm{~cm}^{-3}$ and (b) undoped structure. 
inversely proportional with the barrier layer thickness which increases the distance between the gate and the channel. What is interesting to note is that we have large charge density even in an undoped structure. This is because of the large contribution of the induced polarization (which doesn't depend on $\mathrm{N}_{\mathrm{d}}$ ) to the total sheet charge density. Therefore, it is possible to design AlGaN/GaN HEMT without the need to have a doped barrier.

The magnitude of the threshold voltage (absolute value) is increasing with the increase of barrier thickness as shown in 'figure 2'. This is because we have larger ionized charges and they need more gate voltage to be depleted to the channel.

The relationship between charge density and barrier layer thickness at different doping concentrations is shown in 'figure 3'. The change of charge density with doping concentration at different gate voltage is shown in 'figure 4 '. We notice that the charge density is directly proportional with $\mathrm{d}_{\mathrm{n}}$ and $\mathrm{N}_{\mathrm{d}}$ because the quantity of diffused ionized impurities from the barrier to the charge channel increase with the increase of doping concentration or/and barrier thickness. The slopes of tangents in 'figure 3' for

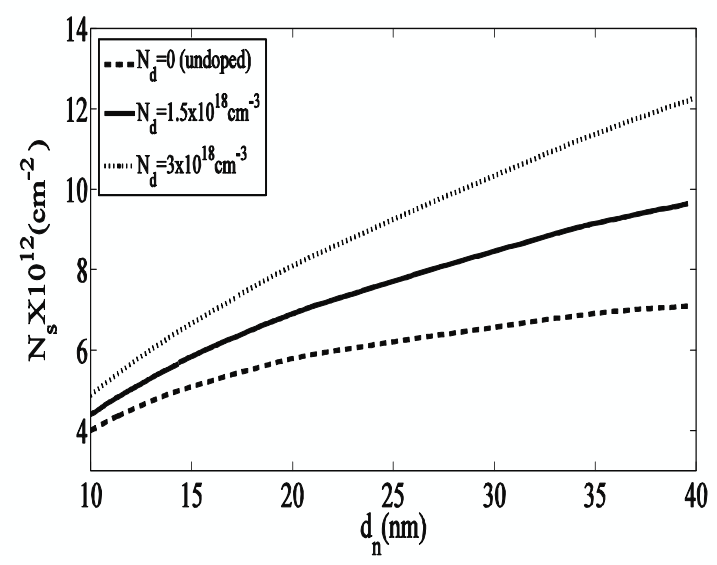

'Figure 3': Sheet charge density vs barrier layer thickness at $\mathrm{x}=0.2$ and $\mathrm{V}_{\mathrm{g}}=0$.

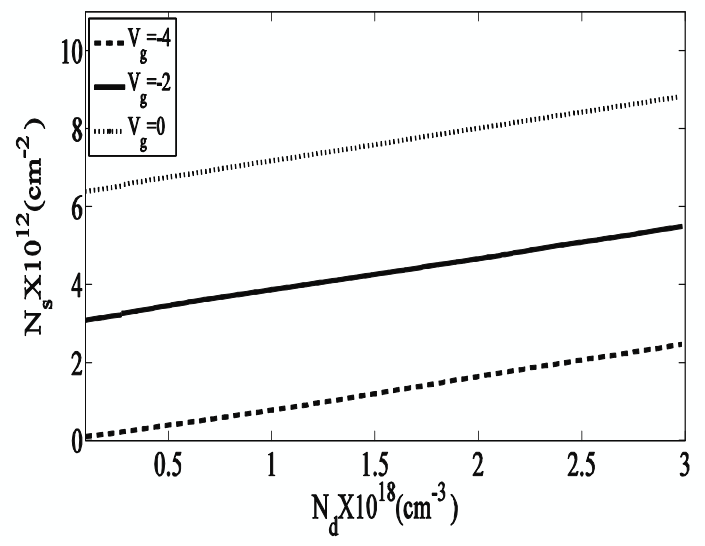

'Figure 4': Sheet charge density vs doping concentration at $x=0.2$ and $\mathrm{d}_{\mathrm{n}}=25 \mathrm{~nm}$.

each curve are larger as $\mathrm{N}_{\mathrm{d}}$ increases. This makes the change of the sheet charge density with barrier thickness more sensitive in case of higher doping density. It is obvious in 'figure 3' that we can get the same value of $\mathrm{N}_{\mathrm{s}}$ by choosing large $\mathrm{d}_{\mathrm{n}}$ with small $\mathrm{N}_{\mathrm{d}}$ or small $\mathrm{d}_{\mathrm{n}}$ with large $\mathrm{N}_{\mathrm{d}}$. The first choice is better 
because it gives lower capacitance and it reduces the effect of deep donors. However, it is important to maintain the barrier thickness $d_{n}$ below the critical value for strain relaxation.

The variation of charge density with Al-mole fraction is shown in 'figure 5'. We include the induced polarization charge $(\sigma)$ in the figure which is higher in case of $\mathrm{AlGaN} / \mathrm{GaN}$ heterostructures compared to other semiconductors. The polarization features a non-linear dependence on $x$. It is increasing directly with $x$ because it depends on the pseudomorphic strain which is a measure of the degree of lattice mismatch between $\mathrm{AlGaN}$ and GaN layers. The lattice mismatch increases with $x$. The sheet charge density increases with $x$ because it depends on $\sigma$ which has the major impact on $\mathrm{N}_{\mathrm{s}}$.

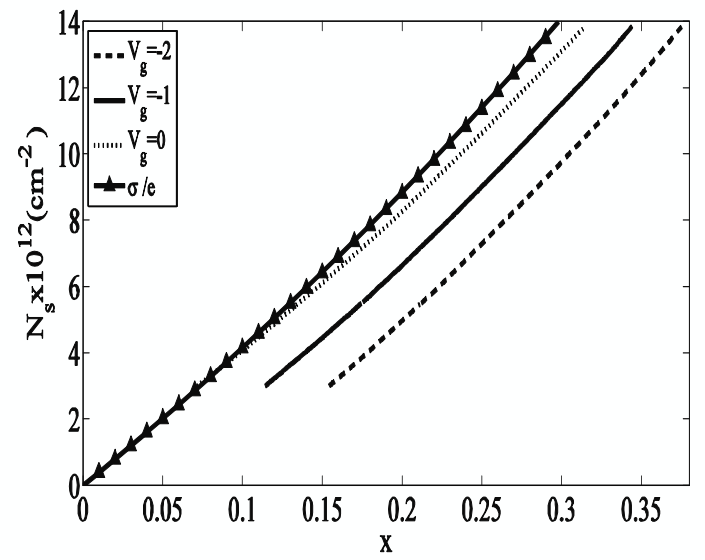

'Figure 5': Sheet charge density vs Al-mole fraction at $d_{n}=25 \mathrm{~nm}$ and $\mathrm{N}_{d}=2 \times 10^{18} \mathrm{~cm}^{-3}$.

We will discuss now the dependance of the plasmon frequency on the variables mentioned above. The impact of gate voltage on the plasmon frequency is shown in 'figure 6'. For a certain HEMT which has been manufactured, its parameters (such as $x, \mathrm{~d}_{\mathrm{n}}$ and $\mathrm{N}_{\mathrm{d}}$ ) can't be changed because they are internal properties. What we can do is the change of $\mathrm{V}_{\mathrm{g}}$ (external parameter) to control the desired value of $\omega_{\mathrm{p}}$. 'Figure 6' shows high sensitivity of the plasmon frequency to gate voltage. For example at $\mathrm{d}_{\mathrm{n}}=15 \mathrm{~nm}$, the sensitivity is $2.98,1.40$ and $0.82 \mathrm{THz} / \mathrm{V}$ at $-4.7<\mathrm{V}_{\mathrm{g}}<-4,-4<\mathrm{V}_{\mathrm{g}}<-2$ and $-2<\mathrm{V}_{\mathrm{g}}<2$, respectively. This demonstrates why the gated plasmons have more practical applications than ungated modes.
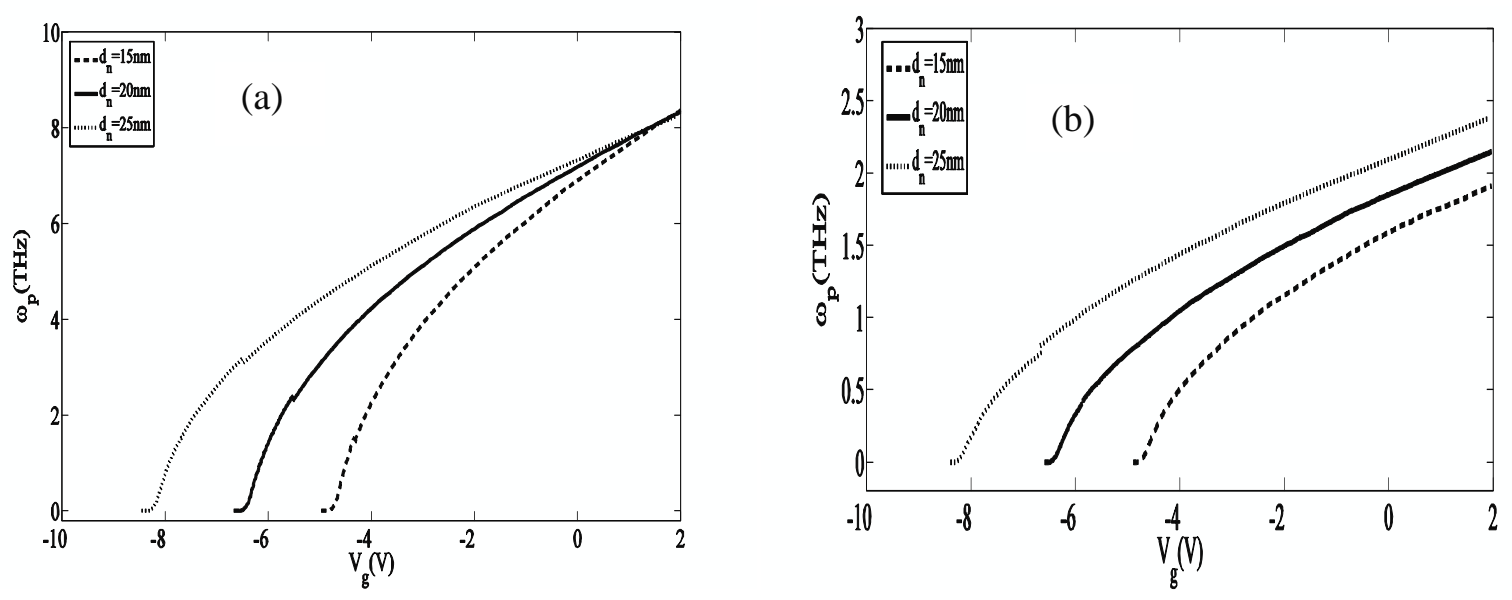

'Figure 6': Plasmon frequency vs gate voltage at $\mathrm{x}=0.3, \mathrm{~N}_{\mathrm{d}}=2 \times 10^{18} \mathrm{~cm}^{-3}$, (a) $\mathrm{L}=60 \mathrm{~nm}$, (b) $\mathrm{L}=500 \mathrm{~nm}$ 
'Figure 7' shows the relation between plasmon frequency and barrier thickness at different doping densities. We notice clearly a maximum value of $\omega_{\mathrm{p}}$ for the undoped case in (a), and it will appear in all curves if we mathematically increase $d_{n}$. This is an interesting optimization which can be obtained by means of the material and geometrical parameters. It is necessary to emphasize that the accuracy of the optimized value is controlled by taking into account the average position $\Delta \mathrm{d}$ of charge density in the channel. Many papers neglect the impact of $\Delta \mathrm{d}$ on $\mathrm{N}_{\mathrm{s}}$ (and as a result on the sensitivity of $\left.\omega_{\mathrm{p}}\right)$. Although $\Delta \mathrm{d}$ is relatively small $(1.7-8.0 \mathrm{~nm})$, it will be considerable when $\mathrm{d}_{\mathrm{n}}$ is small enough. As it is clear in 'equation 13', the charge density depends on the total distance of its position from the gate $\left(=d_{n}+d_{i}+\Delta d\right)$. By increasing $d_{n}, N_{s}$ increases (see 'figure 3 '), while $k$ decreases. Therefore, according to 'equation 2' the effect of $\mathrm{N}_{\mathrm{s}}$ dominates the effect of $\mathrm{k}$ on the Plasmon frequency at values of $d_{n}$ less than critical point, and vice versa. There is a considerable value of $\omega_{p}$ even for undoped heterostructures.
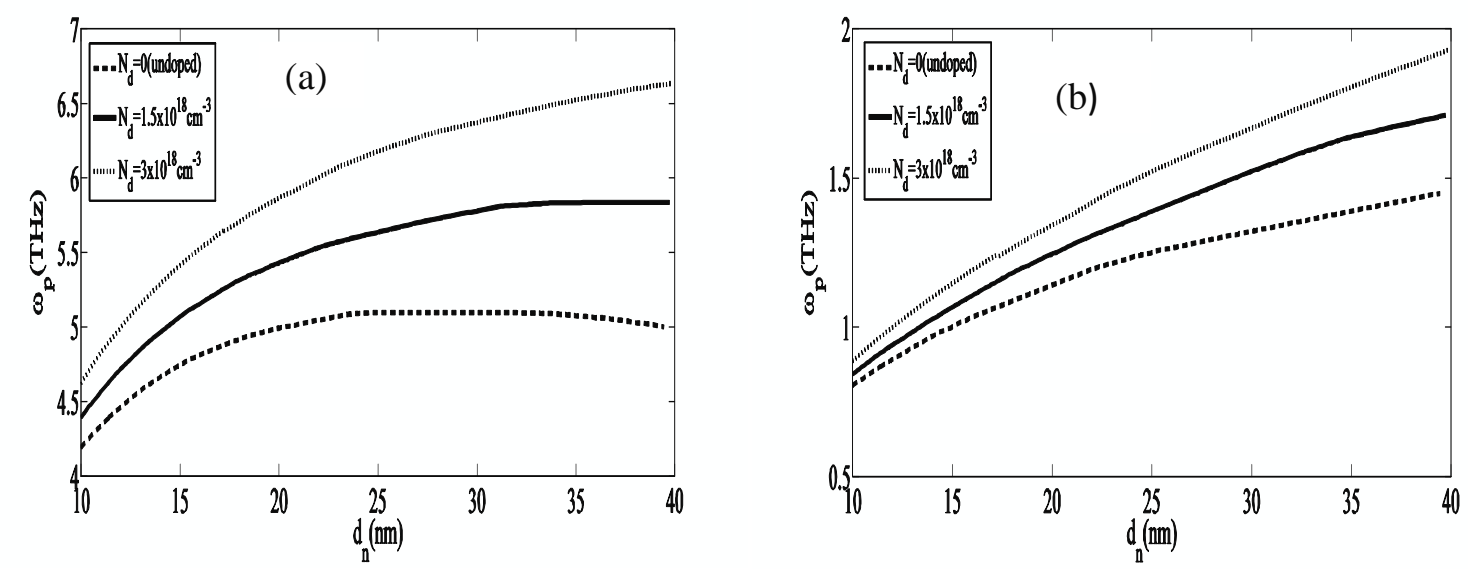

'Figure 7': Plasmon frequency vs barrier thickness at $\mathrm{x}=0.2, \mathrm{~V}_{\mathrm{g}}=0$, (a) $\mathrm{L}=60 \mathrm{~nm}$, (b) $\mathrm{L}=500 \mathrm{~nm}$.

The change of plasmon frequency with doping concentration at different gate voltages is shown in 'figure 8'. We have smaller $\omega_{\mathrm{p}}$ values when $\mathrm{L}=500 \mathrm{~nm}$ than $\mathrm{L}=60 \mathrm{~nm}$ as it appears also in 'figures 6 and 7 .

'Figure 9' represents the dependence of plasmon frequency on the Al-mole fraction in the doped $\mathrm{AlGaN}$ layer at different gate voltages. The value of $\omega_{\mathrm{p}}$ at $\mathrm{L}=60 \mathrm{~nm}$ is approximately twice its value at $\mathrm{L}=500 \mathrm{~nm}$.
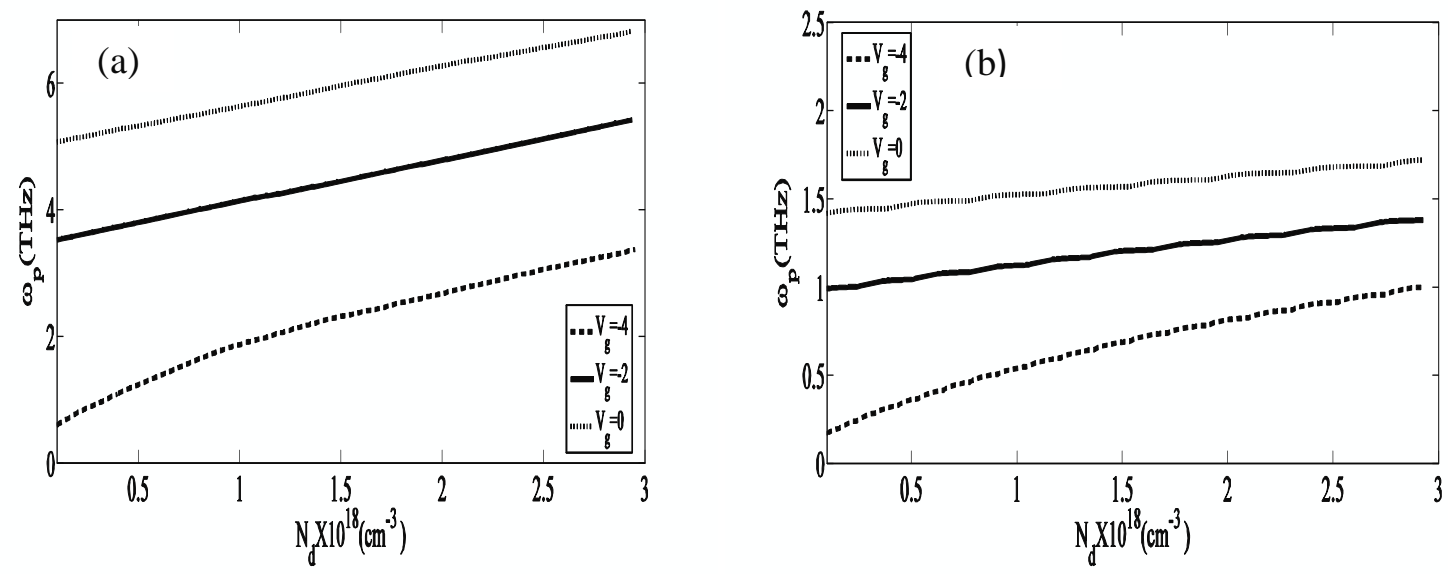

'Figure 8': Plasmon frequency vs doping density at $x=0.2, d_{n}=25 \mathrm{~nm}$, (a) $L=60 \mathrm{~nm}$, (b) $L=500 \mathrm{~nm}$. 

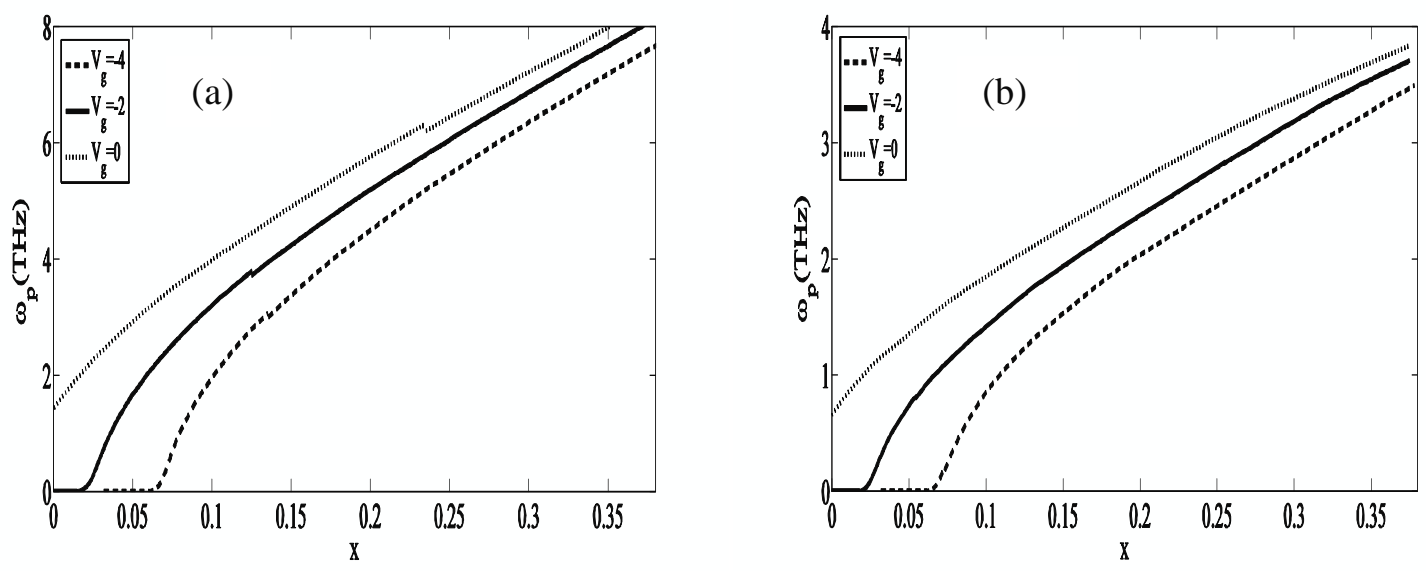

'Figure 9': Plasmon frequency vs Al-mole fraction at $\mathrm{N}_{\mathrm{d}}=2 \times 10^{18} \mathrm{~cm}^{-3}, \mathrm{~d}_{\mathrm{n}}=25 \mathrm{~nm}$, (a) $\mathrm{L}=60 \mathrm{~nm}$, (b) $\mathrm{L}=500 \mathrm{~nm}$.

Finally, the change of plasmon frequency with gate length is shown in 'figure 10'. The plasmon frequency increases with decreasing the gate length, but it should be noted that there are technical limitations in designing a HEMT plasmon device with very short gate length. Also, if the gate length is taken shorter and shorter, the ungated portion of the gate will increase.

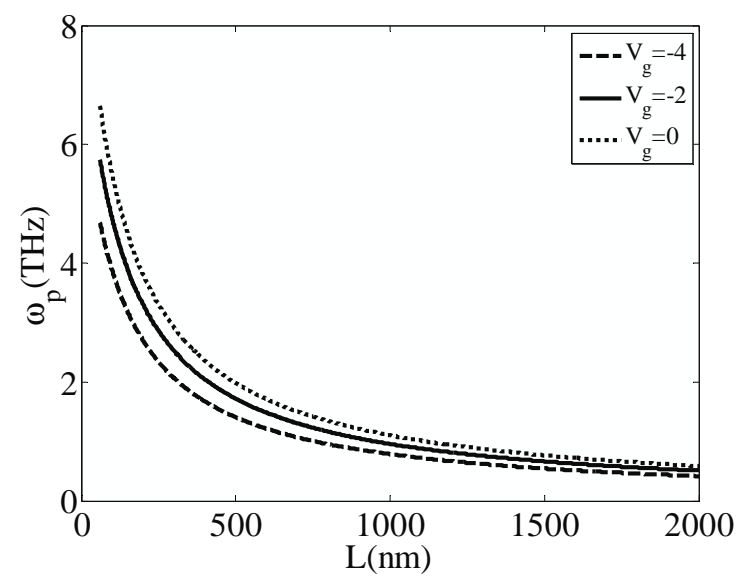

'Figure 10': Plasmon frequency vs gate length at $x=0.25$ and $d_{n}=30$.

The previous calculations of $\omega_{\mathrm{p}}$ are taken for the fundamental plasmon mode $(\mathrm{n}=1)$ in a single-gated HEMT. To get higher frequencies we can consider higher order modes with $n>1$, but in this case, the dipole moment will decrease. It was suggested in [8] that to face this problem, a single-gated device is replaced by field effect transistors arrays.

The 2D plasma frequency can be measured by considering the $\mathrm{THz}$ absorption or emission spectra of a HEMT $[8,26]$. We compare our theoretical results with measurements taken in [26]. The emission absorption was measured for a HEMT of the following parameters: $x=0.3, d_{i}=0, d_{n}=30 \mathrm{~nm}, \mathrm{~N}_{d}=0$, $\mathrm{L}=250 \mathrm{~nm}, \mathrm{~V}_{\mathrm{c}}=4 \mathrm{~V}$. The position of the peaks in the emission spectra represents the plasmon frequency at different gate voltages. We use the same calculation method mentioned above to plot plasmon frequency with gate voltage in "figure 11". The circular points and the error bars at some gate voltages are taken from [26]. We notice a very good agreement of our calculations with experiment measurements. 


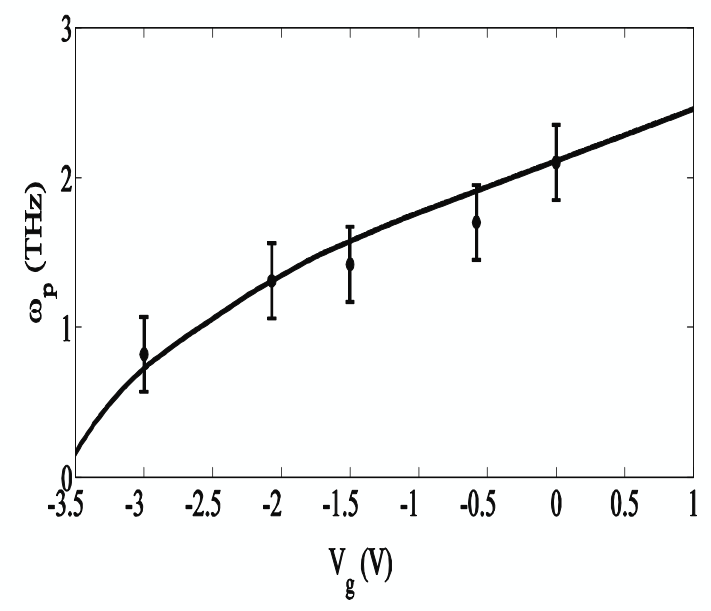

'Figure 11': Theoretical calculations of plasmon frequency vs gate voltage (solid curve) compared to experimental measurements taken from [26] (circular points with error bars: experimental measurements)

\section{Conclusions}

We have calculated the sheet charge density as a function of different transistor design parameters. We have found that the charge density for $\mathrm{AlGaN} / \mathrm{GaN}$ HEMT is so high compared to other semiconductor heterostructures like $\mathrm{AlGaAs} / \mathrm{GaAs}$. This has many technical applications such as biosensors. The charge density increases with $x, \mathrm{~d}_{\mathrm{n}}, \mathrm{N}_{\mathrm{d}}$, and $\mathrm{V}_{\mathrm{g}}$.

The plasmon frequency is directly proportional to $\mathrm{N}_{\mathrm{s}}$. We have found that it is possible to get frequencies from the $\mathrm{GHz}$ band up to $8 \mathrm{THz}$ by choosing suitable HEMT parameters. If we desire to get higher mode frequencies, we should take into account the limitations on all previous parameters and gate length in the practical point of view. The accuracy of the optimized value of the plasmon frequency is controlled by taking into account the average position $\Delta \mathrm{d}$ of charge density in the channel.

We have found high sensitivity of the plasmon frequency to the gate voltage. This is important in fabrication of tunable $\mathrm{THz}$ plasma detectors and other $\mathrm{THz}$ devices. The results encourage using $\mathrm{AlGaN} / \mathrm{GaN}$ heterostructure in such devices as a suitable choice. It is interesting to conclude that the structure produces this spectrum of plasma waves even with an undoped barrier, due to the large induced polarization of the heterostructure.

\section{Acknowledgements}

This work was funded by the EU Commission; the Erasmus Mundus External Cooperation Window (EM ECW) lot 2.

\section{References:}

[1] Rabbaa S, Vandermeiren W and Stiens J 2010 Longitudinal optical phonon-plasmon interaction in Ga-group V compounds for IR and THz applications Proc. of the 15th Annual Symp. of the IEEE Photonics Benelux Chapter (TU Delft: the Netherlands) pp 257-60.

[2] Liu L, Sensale-Rodriguez B, Zhang Z, Zimmermann T, Cao Y, Jena D, Fay P and Xing H 2010 Development of microwave and terahertz detectors utilizing AlN/GaN high electron mobility transistor 21st Int. Symp. on Space Terahertz Technology (Oxford: England) pp 321-25.

[3] Dyakonov M and Shur M 1996 Plasma wave electronics: novel terahertz devices using two dimensional electron fluid IEEE Trans. Electron Devices 43 1640-45.

[4] Mustafa F and Hashim A 2010 Generalization 3D transverse magnetic mode method for analysis of interaction between drifting plasma waves in 2DEG-structured semiconductors and 
electromagnetic space harmonic waves Progress in Electromagnetics Research, PIER 102315 335.

[5] Mustafa F and Hashim A 2010 Plasma wave electronics: a revival towards solid-state terahertz electron devices J. Applied Sc. 10 1352-68.

[6] Popov V, Polischuk O and Shur M 2005 Resonant excitation of plasma oscillations in a partially gated two-dimensional electron layer J. Appl. Phys. 98033510.

[7] Hashim A, Hashizume T, lizuka K and Hasegawa H 2003 Plasma wave interactions in the microwave to $\mathrm{THz}$ range between carriers in a semiconductor 2DEG and interdigital slow waves Superlattice Microst 34 531-37.

[8] Popov V, Shur M, Tsymbalov G and Fateev D 2007 Higher-order plasmon resonances in GaNbased field-effect transistor arrays Int. J. High Speed Electron. Syst 17 557-66.

[9] Hao Y, Yang L and Zhang J 2008 GaN-based semiconductor devices for terahertz Terahertz Sci. Technol 1 51-64.

[10] Ryzhii V, Khmyrova I, Ryzhii M, Satou A, Otsuji T, Mitin V and Shur M 2007 Plasma waves in two-dimensional electron systems and their applications Int. J. High Speed Electron. Syst $17521-$ 38.

[11] Stern F 1967 Polarizability of a 2-dimensional electron gas Phys. Rev. Lett. 18546.

[12] Grimes C and Adams G 1976 Observation of 2-dimensional plasmons and electron-ripplon scattering in a sheet of electrons on liquid-helium Phys. Rev. Lett. 36 145-48.

[13] Allen S, Tsui D and Logan R 1977 Observation of 2-dimensional plasmon in silicon inversion layers Phys. Rev. Lett. 38 980-83.

[14] Hiyamizu S, Saito J and Nanbu K 1983 Improved electron- mobility higher than $106 \mathrm{~cm}^{2} / \mathrm{Vs}$ in selectively doped GaAs/n-AlGaAs heterostructures grown by MBE Jpn. J. Appl. Phys., Part 222 L609-11.

[15] Fiorentini V, Bernardini F and Ambacher O 2002 Pyroelectric evidence of nonlinear macroscopic polarization in III-nitride alloy heterostructures Appl. Phys. Lett. 80 1204-06.

[16] Miller E, Yu E, Poblenz C, Elsass C and Speck J 2002 Direct measurement of the polarization charge in $\mathrm{AlGaN} / \mathrm{GaN}$ heterostructures using capacitance-voltage carrier profiling Appl. Phys. Lett. 80 3551-53.

[17] Ambacher O et al 1999 Two-dimensional electron gases induced by spontaneous and piezoelectric polarization charges in N- and Ga-face AlGaN/GaN heterostructures J. Appl. Phys. 85 3222-33.

[18] Delagebeaudeuf D and Linh N 1982 Metal-(n) AlGaAs-GaAs two-dimensional electron gas FET IEEE Trans. Electron Devices 29 955-60.

[19] Balkanski M and Wallis R 2000 Semiconductor Physics and Applications Oxford University Press Inc. (New York) pp 372-76.

[20] Shur M 1998 GaN based transistors for high power applications Solid State Electron.42 2131-38.

[21] Yahyazadeh R and Hashempour Z 2010 The effect of depletion layer on the cut off frequency of AlGaN/GaN high electron mobility transistors Proc. $27^{\text {th }}$ Int. Conf. in Microelectronics (MIEL) (Nis: Serbia) pp 165-68.

[22] Yakout M, AbdelRassoul R, AbdelFattah A and Essa S 2001 An accurate analytical model of the $\mathrm{AlGaAs} / \mathrm{GaAs}$ high electron mobility transistor (HEMT) $18^{\text {th }}$ National Radio Science Conf. (Mansoura Univ.: Egypt) pp 531-39.

[23] Sen S, Pandey M, Khanna M and Gupta R 1997 A quasi two-dimensional analytical model for threshold voltage of a modulation doped field effect transistor Asia Pacific Microwave Conf. (Hong Kong: China) Vols I-III pp 705-08.

[24] Shey A and Ku W 1989 An analytical current-voltage characteristics model for high electron mobility transistors based on nonlinear charge-control formulation IEEE Trans. Electron Devices 36 2299-306. 
[25] Sun Y, Thompson S and Nishida T 2009 Strain Effect in Semiconductors: Theory and Device Applications Springer (New York) p 150.

[26] El Fatimy A et al $2010 \mathrm{AlGaN} / \mathrm{GaN}$ high electron transistors as a voltage-tunable room temperature terahertz sources J. Appl. Phys. 107024504. 